\title{
1 A plant biodiversity effect resolved to a single genetic locus
}

3 Samuel E. Wuest ${ }^{1,2,3^{*}}$ and Pascal A. Niklaus ${ }^{2}$

4

5 1) Department of Plant and Microbial Biology and Zurich-Basel Plant Science Center, University of

6 Zurich, Zollikerstrasse 107, 8008 Zurich, Switzerland

7 2) Department of Evolutionary Biology and Environmental Studies and Zurich-Basel Plant Science

8 Center, University of Zurich, Winterthurerstrasse 190, 8057 Zurich, Switzerland

9 3) current address: Buchenweg 5, 5436 Würenlos, Switzerland

$11 *$ correspondence to: Samuel E. Wuest (samuel.wuest@ieu.uzh.ch)

\section{Summary}

14 Despite extensive evidence that biodiversity promotes plant community productivity, progress towards understanding the mechanistic basis of this effect remains slow, impeding the development of predictive ecological theory and agricultural applications. Here, we analysed non-additive interactions between genetically divergent Arabidopsis accessions in experimental plant communities. By combining methods from ecology and genetics, we identified a major effect locus

19 at which allelic differences between individuals increases above-ground productivity of

20 communities. In experiments with near-isogenic lines, we show that this diversity effect acts

21 independently of other genomic regions and can be resolved to a single region representing less

22 than $0.3 \%$ of the genome. Using plant-soil-feedback experiments, we also demonstrate that allelic

23 diversity causes genotype-specific soil legacy responses in a consecutive growing period, even after

24 the original community has disappeared. Our work thus suggests that positive diversity effects can

25 be linked to single Mendelian factors, and that a range of complex community properties can have a 
26 simple cause. This may pave the way to a better understanding of diversity effects, and to novel

27 breeding strategies, focussing on phenotypic properties that manifest themselves beyond isolated

28 individuals, i.e. at a higher level of biological organisation.

\section{Main Text}

31 More than two decades of plant ecological research and the publication of hundreds of studies have

32 firmly established that positive biodiversity effects, in particular on community yield, are the rule

33 rather than exception and are often substantial ${ }^{1,2}$. These positive effects of biological diversity on

34 community functioning have been explained by larger community-level resource use promoted by niche complementarity, facilitation, or by reduced negative density-dependent effects of enemies ${ }^{3-5}$.

Yet our understanding of the particular driving mechanisms remains poor, for several reasons. First, diversity effects are emergent properties that only manifest in comparisons of communities differing in diversity ${ }^{6}$. Second, diversity effects, and the mechanisms that drive these, may change with environmental conditions ${ }^{7,8}$. Third, while there is no doubt that functional trait differences underly biodiversity effects ${ }^{9}$, trait-based analyses remain to some degree phenomenological because evolutionary forces have led to the formation of trait syndromes, i.e. to sets of highly correlated traits that reflect fundamental trade-offs between ecological strategies ${ }^{10,11}$. The observed variation in traits thus is confounded with evolutionary history ${ }^{12}$, and it remains almost impossible to distinguish

44 the traits that are true drivers of biodiversity effects from traits that are merely correlated. It therefore remains difficult to develop predictive ecological theory and apply it, for example, in breeding and agriculture.

Most biodiversity research to date has focused on variation among species, but experimental ${ }^{13}$,

48 theoretical $^{14}$, and observational ${ }^{15}$ studies have shown that positive diversity effects on productivity

49 also occur at levels of organization above (e.g. landscape level) and below species (e.g. genotype

50 level). A substantial part of the trait variation apparent in plant communities occurs within species ${ }^{16}$, 
51 and increased intra-specific variation can have similar effects as inter-specific trait variation in low-

52 diversity systems $s^{13,17,18}$. Despite qualitative differences, there may therefore be commonalities of

53 trait variation within and between species with respect to effects and mechanisms, indicating that

54 studies at the genotype level may provide some insights into effects of species-level variation and

55 vice versa. A methodological advantage of intra-specific biodiversity studies is that genetic methods

56 can circumvent some of the problems encountered in species-level diversity studies. Specifically,

57 crosses between genotypes allow trait variation among individuals to be re-arranged ${ }^{19}$ without

58 confounding with population structure or differentiation into ecological strategies. However, genetic

59 approaches are normally used to study properties of individuals rather than emergent properties of

60 communities. Here, we demonstrate in a case study how the genetic approach can be harnessed to

61 identify the genetic underpinnings of biodiversity effects.

\section{Results}

64 Using model plant communities (Fig. 1a), we screened ten pair-wise mixtures of genetically divergent natural accessions of Arabidopsis thaliana for which mapping populations are publicly available (Methods). Mixture communities of the two genetically divergent accessions Bayreuth (Bay) and Shadhara (Sha) reproducibly exhibited positive net biodiversity effects, i.e. mixtures

68 produced a higher community-level shoot biomass than the average of their monocultures. This

69 depended on soil conditions, with effects that were essentially absent on peat-rich soil but grew to

70 an overyielding of $16 \%$ with increasing amounts of sand in the substrate (sand content $\times$ diversity:

$71 \mathrm{~F}_{1,160}=4.57$, ANOVA $\mathrm{P}<0.05$; Fig. $1 \mathrm{~b}$ and Supplementary Figure 1a). Analysis by additive 72 partitioning ${ }^{6}$ revealed that these community-level biodiversity effects were due to complementarity 73 rather than selection effects (sand content $\times$ complementarity effect, $\mathrm{F}_{1,77}=7.21$, ANOVA P $<0.01$;

74 Supplementary Figure 1b). Specifically, in our study both accessions benefited from growing in 75 mixed communities. 
76 To analyse the genetic basis of the positive diversity effect in mixed Bay-Sha communities, we

77 performed quantitative trait locus (QTL) mapping using publicly available recombinant inbred lines

78 (RILs). These RILs are largely homozygous (Fig. 1c) and have been derived from a cross between

79 the Bay and Sha accessions, followed by multiple subsequent rounds of selfing ${ }^{20}$. For efficient

80 mapping, we capitalized on a so called competition diallel. Traditional diallel designs systematically

81 cross sets of parental lines realizing all possible combinations and are used in breeding to determine

82 the genetic basis of traits; specifically, diallel analysis partitions the trait variation of crosses into

83 additive contributions ${ }^{21}$ of parental lines (general combining abilities; GCA) and cross-specific

84 effects (specific combining abilities; SCA), with the latter interpreted as consequences of

85 dominance or epistasis. By substituting individuals and crosses with communities and mixtures, the

86 principle of diallels can be applied to the analyses of biodiversity effects in communities ${ }^{22}$, which

87 we did here (Fig. 1c, d). In this context, the distinction between maternal and paternal effects ceases

88 to apply, simplifying the design to a half-diallel. SCAs then quantify the deviations of mixture

89 yields from expectations based on additive average contributions of the two genotypes. We

90 combined 18 RILs and the two parental accessions Bay and Sha in a diallel, in four replicate blocks,

91 on sand-rich soil. We detected significant positive genotype diversity effects on above-ground

92 biomass production (Fig. 2a, $\mathrm{F}_{1,189}=10.47, \mathrm{P}<0.01$ ), indicating that the traits that promote

93 biodiversity effects are heritable. As expected, a large proportion of the variation in SCA remained

94 unexplained. We therefore tested for allelic diversity effects on SCAs at 69 marker positions. Both a

95 marker regression technique and a standard QTL procedure revealed a major effect locus on the

96 lower arm of chromosome four where allelic diversity at the community level resulted in higher

97 SCAs (Fig. 2b and Supplementary Figure 2).

98

99 
100 With 18 recombinant lines, mapping resolution was limited and other effect loci or genetic

101 interactions among loci may have gone unnoticed. We thus aimed at resolving the allelic diversity

102 effect further to a single Mendelian factor. For this, we isolated a family of 19 near isogenic lines

103 (NILs) that genetically varied only on the lower arm of chromosome 4, and in which we selected

104 and inferred further recombination events by molecular markers and whole-genome re-sequencing

105 (Fig. 3a,b, Supplementary Figure 3). With these NILs, we performed a second diallel experiment,

106 replicated once on peat-rich soil where we expected no diversity effects and once on sand-rich soil

107 where we expected positive diversity effects. Indeed, no locus was associated with positive allelic

108 diversity effects on above-ground biomass on peat-rich soil (Fig. 3c). In contrast, on sand-rich soil

109 we found a positive allelic diversity effects at a single locus (overyielding of 4.5\%, Fig. 3d, P <

110 0.01), represented by a region of approx. $310 \mathrm{~kb}$ in size (termed locus Chr4@16.92: between 16.92

111 to $17.23 \mathrm{Mb}$ ). The overyielding of allelic mixtures of otherwise isogenic lines was transgressive:

112 communities that contained individuals carrying different alleles at locus Chr4@16.92 in the NIL

113 diallel produced more biomass than the most productive mono-allelic community $(\mathrm{t}=2.32 ; \mathrm{P}=$

114 0.02), suggesting some form of functional complementarity between genotypes: relating to, without

115 reference to a specific mechanisms at play, a phenomenon of two alleles positively interacting with

116 each other when distributed amongst homozygous individuals of a community. Using structural

117 equation models, we tested whether these allelic diversity effects were related to observed

118 genotypic differences in shoot phenology or disease symptoms, but this was not the case

119 (Supplementary Figure 4). Interestingly, however, this analysis showed that allelic diversity already

120 manifested itself in increased community-level leaf cover early in the experient, when plants just

121 started to produce flowering bolts (Supplementary Figure 4), indicating that the effect persisted

122 through time. The allelic diversity effect we found, could, in principle, strictly depend on the

123 genetic background. To rule out this possibility, we compared monocultures and mixtures of a

124 second, independent pair of near-isogenic lines on both peat-rich or sand-rich soil (Supplementary 
125 Figure 5). Again, we found a significant allelic diversity effect on above-ground biomass that 126 depended on soil $\left(\mathrm{F}_{1,164}=4.17\right.$, ANOVA $\mathrm{P}=0.04$ for soil $\mathrm{x}$ allelic diversity; $5.4 \%$ net overyielding

127 on sand), and a significant effect of soil on the complementarity effect $\left(\mathrm{F}_{1,82}=4.8, \mathrm{P}=0.03\right)$.

128 In conclusion, two consecutive diallel experiments using only 37 recombinant lines were sufficient 129 to resolve a plant biodiversity effect to a genomic region representing $2.5 \%$ of the Arabidopsis 130 genome (containing approx. 86 genes), which emphasizes the extreme efficiency of our approach.

131 Our work suggests that biodiversity effects between genotypes can be dissected into discrete genetic 132 elements that have major additive contributions.

134 There is growing evidence that productivity responses in plant biodiversity experiments ${ }^{4,5,23,24}$ and 135 their increase through time ${ }^{25}$ are related to diversity-dependent soil conditioning. To test whether 136 allelic diversity causes effects through soil conditioning also in our study, we performed soil 137 feedback experiments. Our objectives were twofold: first, we were interested whether there were 138 general effects of allelic diversity on soil quality; second, we aimed to test whether allelic 139 interactions occurred among plants that did not grow simultaneously, i.e. whether allelic effects 140 were mediated through time by soil legacy. We assessed soil conditioning by growing indicator 141 plants ("phytometers”"25) on soil collected from both diallel experiments ${ }^{26}$ when these were harvested 142 (Fig. 1d and Supplementary Discussion). The phytometers were the two parental accessions Bay or 143 Sha for the RIL diallel, and two near-isogenic lines in the NIL diallel.

144 We indeed found phytometer-specific soil legacy responses that depended on the allelic diversity of 145 the communities that had conditioned the soils in the preceeding growing period (Fig. 4; RIL 146 diallel: diversity at marker MSAT4.9 $\times$ phytometer; $F_{1,166}=6.48$; ANOVA P $=0.012$; NIL diallel: 147 diversity at locus Chr4@16.92 × phytometer; $\mathrm{F}_{1,168}=5.61 ; \mathrm{P}=0.02$; Supplementary Table 1; 148 Supplementary Figure 6). These phytometer-specific responses to soil legacy were independent of 149 differences in previous community productivity and associated resource depletion (the effects 
remained statistically significant and comparable in size when first adjusting for community biomass in linear models). However, the effects differed between phytometers and experiments, i.e. they depended on environmental or genetic context (Supplementary Discussion). This is not surprising in light of the complex mechanisms involved. Developing a full understanding of the biological mechanisms at play will thus require further experiments, including soil analyses.

155 Nonetheless, these experiments demonstrate that allelic differences at a single QTL cause interactions between individuals within a community and also, mediated by a soil-borne factor, through time. The latter can be perceived as an "extended phenotype” sensu Dawkins ${ }^{27}$, the expression of which depends on interactions between group members. We were intrigued to find that we could, in principle, have genetically mapped the allelic diversity effect solely through its soil legacy; in other words, by QTL mapping this extended phenotypic property of allelic mixtures (Supplementary Figure 6e,f; Supplementary Discussion).

\section{Discussion}

164 Our study systematically resolves a biodiversity effect, once identified in a specific set of interacting plants and environmental context, to between-individual allelic differences in a single chromsomal region So far, complex emergent properties of plant communities did not necessarily seem genetically tractable, especially since quantitative traits of individuals often are polygenic ${ }^{28}$ if

168 not omnigenic in nature ${ }^{29}$. A single case study obviously is limited with respect to generalizations,

169 but we consider it possible that in many cases between-individual allelic complementarity and resulting biodiversity effects might instead have a relatively simple genetic architecture - a feature

171 not uncommon for other types of biotic interactions ${ }^{30}$. Our genetic approach is extensible to the 172 study of interactions among other genotype combinations, and, with modifications, among species, 173 and could thus lead to fundamental new insights into the traits and genetic underpinnings of 174 biodiversity effects in more natural systems. Equally importantly, the genetic tractability of such 
175 effects may allow efficient breeding of genotype mixtures that support increased yields through

176 some form of functional complementarity while maintaining low variation in economically relevant

177 traits. Biodiversity effects have received relatively little attention in breeding and conventional

178 agriculture, with the notable exception of crop rotation ${ }^{31}$ and intercropping of cultivars and

179 species $^{32-34}$. Instead, sustaining a growing global human population heavily depends on increasing

180 nutrient inputs to crop production systems ${ }^{35}$, on breeding of single genotypes for monoculture

181 performance $^{36}$, and on the use of within-individual diversity effects termed heterosis ${ }^{37}$. Our

182 approach might help bypass constraints imposed on the performance of single genotypes, by

183 shifting breeding efforts from the individual to the system level ${ }^{38}$.

185 Methods

186 Germplasm

187 The Shadhara and Bayreuth accessions were kindly provided by Nuno Pires (University of Zurich)

188 and had originally been obtained from the Nottingham Arabidopsis stock center (NASC). We

189 selected these two genotypes following an initial screen of ten pairs of accessions, because they

190 exhibited an above-average but not extreme biodiversity effect when growh together on sand-rich

191 soil, and because a high-quality and frequently used RIL population is publicly available ${ }^{20}$. It also

192 was interesting that the biodiversity effect vanished on peat-rich soil. The 18 RILs (representing the

193 "RIL-minimal set" and line 33RV191 used to generate NILs (all contained in the core-pop set of

194165 lines) were ordered from the Versailles Arabidopsis stock center

195 (http://publiclines.versailles.inra.fr) and propagated in a growth chamber. A Bay $\times$ Sha RIL

196 (33RV191) was confirmed to be heterozygous at two PCR marker positions on chromosome 4

197 (Table S2). Upon selfing of this line, the two NILs 33RV191-Sha and 33RV191-Bay were isolated

198 (referred to as NIL-Bay or NIL-Sha hereafter), and their genomes were re-sequenced as described

199 below. Using the same procedure, an independent pair of near-isogenic lines was derived upon 
selfing the Bay×Sha RIL 33RV77, giving rise to NILs 33RV77-Sha and 33RV77-Bay. Furthermore,

201 after selfing of another single heterozygous $F_{10}$ individual of line 33RV191, we screened 160

202 offspring for recombination between the ShaBa5, ShaBa6 and ShaBa8 markers on chromosome

203 four. Upon selfing of 23 putative recombinant offspring, we isolated 19 homozygous recombinant

204 lines for which we confirmed a recombination event in the region by PCR. We then performed

205 whole-genome re-sequencing to confirm the isogenic background and to infer recombination

206 breakpoints for this heterogeneous inbred family (referred to as NILs throughout the text) as 207 described below.

$\underline{\text { Soils and growth conditions }}$

210 Soils consisted of different mixtures of a peat and nutrient rich soil (Einheitserde ED73; $\mathrm{pH} \sim 5.8, \mathrm{~N}$

$211250 \mathrm{mg} \mathrm{L}^{-1} ; \mathrm{P}_{2} \mathrm{O}_{5} 300 \mathrm{mg} \mathrm{L}{ }^{-1} ; 75 \%$ organic matter content; Gebrüder Patzer GmbH, Sinntal-Jossa,

212 Germany) and finely grained quartz sand. Pot for all mixture experiments were $7 \times 7 \times 8 \mathrm{~cm}$ in size.

213 The experiment using the parental lines Bay and Sha was replicated on a soil quality gradient with

214 sand contents of $0 \%, 40 \%, 75 \%$ and $80 \%$, which resulted in a near-linear decrease of pot-

215 productivity from the highest to lowest ED73 content, likely through a dilution of soil nutrients

216 (Fig. S1). For the rough-mapping of the diversity effect using RILs, we used a mixture of $80 \%$ sand

217 and 20\% ED73. For the fine-mapping diallel using NILs, we used either a $80 \%: 20 \%$ or a 20\%:80\%

218 sand:ED73 mixture.

219 Seeds were sown directly onto soils (approx. 10 seeds per position, 4 positions per pot, Fig. 1a).

220 The pots were placed in growth chambers or greenhouse compartments and covered with plastic

221 lids to maintain a high humidity for germination and initial seedling establishment. Additional light

222 was provided if necessery, achieving a photoperiod of 14-16 hours. Day-time and night-time 223 temperatures were maintained around $20-25^{\circ} \mathrm{C}$ and $16-20{ }^{\circ} \mathrm{C}$, respectively. Seedlings were thinned 224 continuously until a single healthy seedling remained per position. 
225 Once seedlings were established, the pots were placed in a greenhouse compartment with automated

226 watering (every 2 days). In summer 2015, daytime temperatures were extremely high, and the first

227 block of the RIL diallel was therefore grown in a growth chamber with full climate control $(8 \mathrm{~h}$

228 night $/ 16 \mathrm{~h}$ day; $60 \%$ humidity; $18 / 23^{\circ} \mathrm{C}$ night/day temperature). The second block was grown in the

229 growth chamber for a month before it was re-located to the regular greenhouse compartment.

230 Pots that did not contain all four originally planted individuals were discarded. Plants were

231 harvested 43-51 days after sowing, with the specific harvest date determined by the occurence of

232 approx. 5-10 dehiscent siliques on the earliest flowering genotypes within a block.

233 After the diallels were harvested, soil feedback trials were established by dividing the soil of a pot

234 into two smaller pots $5.5 \times 5.5 \times 6.0 \mathrm{~cm}$ in size. The respective phytometers (Bay or Sha for the RIL 235 diallel, 33RV191-Sha or 33RV191-Bay for the NIL diallel) were sown directly onto the soil. Again, 236 seeds were oversown and seedlings thinned continously until a single healthy individual remained.

237 Phytometer experiments were harvested either 36 days after sowing (peat-rich soil remaining after 238 the NIL diallel, harvested early because plant roots started to grow out of the pots) or 49-58 days 239 after sowing (sand-rich soil, each block was harvested on a single day). For all experiments, the 240 position of the individual pots was randomized across trays during seedling establishment, and 241 across watering tables after seedling establishment. Throughout the experiment, pots were re242 positioned randomly within trays and tables every 7-10 days. Pots were watered ad libitum, and in 243 case of high population densities of dark-winged fungus gnats, the systemic insectizide ActaraG 244 (Syngenta Agro AG) was applied according to the manufacturers recommendation. After 245 harvesting, plant biomass was dried at $65^{\circ} \mathrm{C}$ for at least three days before weighing. We determined 246 early rosette cover in the NIL diallel by photographing pots 27 days after sowing and estimating the 247 horizontally projected community-level rosette area using the Easy Leaf Area software ${ }^{39}$. We 248 further recorded the occurrence of leaf disease symptoms (wilting, blotching, or early senescence) 24930 days after sowing. As a proxy for flowering time in the NIL diallel, we measured flowering bolt 
250 height of all plants 35 days after sowing; by then, $>98 \%$ of the individuals had a flowering bolt

251 longer than $0.5 \mathrm{~cm}$ ). The NIL diallel was harvested 50 days after sowing.

253 Experimental designs

254 To test the soil-dependency of biodiversity effects in mixed Shadhara-Bayreuth communities, four

255 soil substrates varying in sand content were prepared as described above. We then grew 12 replicate

256 monocultures of each accession plus 24 replicate mixtures per soil type (total of $48 \times 4=192$ pots).

257 The RIL diallel consisted of a half diallel replicated in four blocks. All pair-wise RIL combinations

258 were realized once per block except for RIL monocultures which were replicated twice. For the

259 follow-up soil feedback experiment, we re-used soil from only the first two blocks of the diallel. We

260 re-mixed the soil of each single pot after harvesting the plants, and re-distributed it into two smaller

261 plots that were sown with either a Shadhara or Bayreuth parental genotype that served as 262 phytometers.

263 The NIL diallel used for fine-mapping was realized in a single block that contained all pair-wise

264 combinations of the 19 NILs including monocultures. The subsequent soil feedback part of the 265 experiment was realized as described for the RIL diallel, using either 33RV191-Bay or 33RV191-

266 Sha genotypes as phytometers. To test if the allelic diversity effect was strictly dependent on genetic 267 background, we grew 21 replicate monocultures of each NIL 33RV77-Bay or 33RV77-Sha, plus 42 268 replicate mixtures, on either peat-rich (80\% ED73, 20\% sand) or sand-rich $(20 \%$ ED73, $80 \%$ sand) 269 soil (total of $42 \times 2 \times 2=168$ pots).

271 Genotyping and line re-sequencing

272 PCR-based genotyping assays (Table S2) were developed based on deletions in the Sha genome as 273 predicted by the Polymorph tool (http://polymorph.weigelworld.org) ${ }^{40}$. 
274 Barcoded libraries for genome re-sequencing were prepared using the Illumina Nextera DNA

275 Library Prep Kit (FC-121-1031, Illumina Inc. San Diego, CA) in combination with the Nextera

276 Index Kit (96 indices, FC-121-1012) and pair-end sequenced on an Illumina HiSeq 2500 (2x150 bp,

277 rapid run). The clustering and sequencing were performed at the Functional Genomics Center

278 Zurich. Sequences were aligned to the Arabidopsis genome (Col-0 genome, TAIR version 10) using

279 BWA $^{41}$, aligned read sorting and variant calling were performed using samtools ${ }^{42}$. Aligned genomic

280 sequences of the parental accessions Bay-0 and Sha were downloaded from the 1001 genomes

281 project data center (http://1001genomes.org). The VCF-file produced by the samtools software was

282 loaded into the R Statistical Software ${ }^{43}$, where the subsequent analyses were performed: variant

283 calls were filtered (for differences in genotype calls between the Sha and Bay genomes, quality of

284 variant calls, population-level minimal minor allele frequency 0.2 ; maximum heterozygosity 0.2 ).

285 Inference of genotype calls at polymorphic sites was performed as described previously ${ }^{44}$ and

286 inference of parental alleles was improved using functionality implemented in the MPR package ${ }^{44}$.

287 Genotype reconstruction was then performed in $\mathrm{R}$ using a simple hidden Markov model as

288 implemented in the R package HMM, with hidden state starting probabilities (Bay, Het or Sha) all

289 set to $1 / 3$, and transition probabilities from one state to itself set to 0.99998 and to the other two

290 states set to 0.00001 each. Emission probabilities of genotype calls given a state, e.g. Bay, were set

291 to $0.35,0.25,0.25,0.15$ for genotypes calls Bay, Het, Sha or missing, etc.

\section{$\underline{\text { Statistical analyses }}$}

294 We analyzed data from the diallel experiments using linear mixed models summarized by analysis 295 of variance (ANOVA). The model terms included, in this order, the general combining abilities 296 (GCA) of genotypes (a factor with 20 levels in the RIL diallel and 19 levels in the NIL diallel), the genotype diversity in the pot (GD, 1 or 2 genotypes), the allele identity in the genotype 
Bay/Bay] or 2 [Sha/Bay]), and the genotype composition planted in the pot (comp). The factor GCA was created by superimposing the model matrices for factors coding for the first and second genotype (factors with 20 and 19 levels for RIL and NIL diallels, respectively). The significance of GD, A, and AD were determined using F-tests with comp as error term (denominator). A and AD were encoded in such a way that these contrasts applied only to genotype monocultures and mixtures, respectively. Technically, this was achieved by including a third level in the factor that did not vary in the other group. Fitting A and AD after GD therefore only explained variance in these subsets. The diallel model was extended by additional terms and the corresponding interactions when these applied; specifically, the RIL diallel included a block effect. The NIL diallel included terms for soil type, and interactions of all the terms above with soil type (for example, soil×AD was tested using soil $\times$ comp as error term). The soil feedback experiments included further interactions with phytometer (RIL and NIL diallel), and phytometer $\times$ soil (NIL diallel). Effects of pot biomass in the diallel (diallel biomass and diallel biomass $\times$ soil) were accounted for in these linear models, and data were square-root transformed to obtain normally distributed residuals. Specific combining abilities for mapping were calculated directly, within blocks, by solving the

314 linear model $\mathrm{m}=\mathrm{X}$ GCA + SCA where $\mathrm{X}$ is the design matrix describing the genotype composition of a pot. Monoculture SCAs were also determined but not used for QTL mapping of allelic diversity within RIL mixtures. In the RIL diallel, the SCAs of each genotype composition was first calculated per block and then aggregated over all blocks using least-square estimates. Marker regression was performed contrasting SCAs of mono-allelic RIL mixtures ("BB" and "SS" compositions) with mixed-allelic mixtures ("BS" compositions) using the glht-function provided by the multcomp package $^{43}$. QTL mapping was also performed using the $\mathrm{R} / \mathrm{qtl}$ package and interval mapping (scanone-function), with both mono-allelic compositions at a given locus re-coded as to the same level ("mono-allelic") and compared against mixed-allelic compositions. Genome-wide significance was assessed by resampling $(n=5000)$. 
324 To test the relationships of allelic diversity effects on SCAs with measured traits, we developed

325 multip-group (sand-rich and peat-rich soil) structural equation models using lavaan

326 (http://lavaan.ugent.be). Allelic diversity at Chr4@16.92 was included as exogenous variable;

327 endogeneous variables were two metrics of trait variation among genotypes (which are possible

328 indicators of complementarity), and early community-level projected leaf area (see above). Trait

329 variation was quantified as difference among bolt length of the genotypes (square-root-

330 transformed), and as difference in the occurence of leaf disease symptoms (a binary variable).

331 Starting with a near-saturated model, the modelled paths were simplified in an educated way until a

332 minimal model was found for which the model-implied and observed covariance structure among

333 variables did not differ significantly $\left(\mathrm{X}^{2}\right.$-test $)$.

\section{References}

336 1. Cardinale, B. J. et al. The functional role of producer diversity in ecosystems. Am. J. Bot. 98, 572-592 (2011).

2. O'Connor, M. I. et al. A general biodiversity-function relationship is mediated by trophic level. Oikos 126, 18-31 (2017).

340 3. Cardinale, B. J., Palmer, M. A. \& Collins, S. L. Species diversity enhances ecosystem

7. Ratcliffe, S. et al. Biodiversity and ecosystem functioning relations in European forests

8. Tylianakis, J. M. et al. Resource heterogeneity moderates the biodiversity-function 
10. Wright, I. J. et al. The worldwide leaf economics spectrum. Nature 428, 821-827 (2004).

11. Díaz, S. et al. The global spectrum of plant form and function. Nature 529, 167-171 (2015).

12. Cadotte, M. W., Cardinale, B. J. \& Oakley, T. H. Evolutionary history and the effect of biodiversity on plant productivity. Proc. Natl. Acad. Sci. USA 105, 17012-17017 (2008).

13. Crutsinger, G. M. et al. Plant genotypic diversity predicts community structure and governs an ecosystem process. Science 313, 966-968 (2006).

14. Wang, S. \& Loreau, M. Ecosystem stability in space: $\alpha, \beta$ and $\gamma$ variability. Ecol. Lett. 17, 891-901 (2014).

15. Oehri, J., Schmid, B., Schaepman-Strub, G. \& Niklaus, P. A. Biodiversity promotes primary

16. Siefert, A. et al. A global meta-analysis of the relative extent of intraspecific trait variation in

17. Crutsinger, G. M., Souza, L. \& Sanders, N. J. Intraspecific diversity and dominant genotypes

18. Prieto, I. et al. Complementary effects of species and genetic diversity on productivity and

19. Mendel, G. Versuche über Pflanzenhybriden. in Verhandlungen des naturforschenden

20. Loudet, O., Chaillou, S., Camilleri, C., Bouchez, D. \& Daniel-Vedele, F. Bay-0 x Shahdara

21. Griffing, B. Concept of General and Specific Combining Ability in Relation to Diallel

22. Griffing, B. Genetic-Analysis of Plant Mixtures. Genetics 122, 943-956 (1989).

23. Zak, D. R., Holmes, W. E., White, D. C., Peacock, A. D. \& Tilman, D. Plant diversity, soil microbial communities, and ecosystem function: Are there any links? Ecology 84, 20422050 (2003).

24. Bukowski, A. R. \& Petermann, J. S. Intraspecific plant-soil feedback and intraspecific overyielding in Arabidopsis thaliana. Ecol. Evol. 4, 2533-2545 (2014).

26. Clements, F. E. \& Goldsmith, G. W. The Phytometer Method in Ecology. (Carnegie

27. Dawkins, R. The Extended Phenotype. (Oxford University Press, 1982). 
28. MacKay, T. F. C., Stone, E. A. \& Ayroles, J. F. The genetics of quantitative traits: Challenges and prospects. Nature Reviews Genetics 10, 565-577 (2009).

29. Boyle, E. A., Li, Y. I. \& Pritchard, J. K. An Expanded View of Complex Traits: From Polygenic to Omnigenic. Cell 169, 1177-1186 (2017).

30. Wilfert, L. \& Schmid-Hempel, P. The genetic architecture of susceptibility to parasites. BMC Evol. Biol. 8, 187 (2008).

31. Wittwer, R. A., Dorn, B., Jossi, W. \& Van Der Heijden, M. G. A. Cover crops support ecological intensification of arable cropping systems. Sci. Rep. 7, 41911 (2017).

32. Finckh, M. R. et al. Cereal variety and species mixtures in practice, with emphasis on disease resistance. Agronomie 20, 813-837 (2000).

33. Zhu, Y. et al. Genetic diversity and disease control in rice. Nature 406, 718-722 (2000).

34. Litrico, I. \& Violle, C. Diversity in Plant Breeding: A New Conceptual Framework. Trends in Plant Science 20, 604-613 (2015).

35. Tilman, D., Cassman, K. G., Matson, P. A., Naylor, R. \& Polasky, S. Agricultural sustainability and intensive production practices. Nature 418, 671-677 (2002).

36. Edgerton, M. D. Increasing crop productivity to meet global needs for feed, food, and fuel. Plant Physiol. 149, 7-13 (2009).

37. Lippman, Z. B. \& Zamir, D. Heterosis: revisiting the magic. Trends in Genetics 23, 60-66 (2007).

38. Weiner, J., Du, Y. L., Zhang, C., Qin, X. L. \& Li, F. M. Evolutionary agroecology: individual fitness and population yield in wheat (Triticum aestivum). Ecology 98, 2261-2266 (2017).

39. Easlon, H. M. \& Bloom, A. J. Easy Leaf Area: Automated Digital Image Analysis for Rapid and Accurate Measurement of Leaf Area. Appl. Plant Sci. 2, apps.1400033 (2014).

40. Fitz, J. et al. personal communication. http://polymorph.weigelworld.org

41. Li, H. \& Durbin, R. Fast and accurate short read alignment with Burrows - Wheeler transform. Bioinformatics 25, 1754-1760 (2009).

42. Li, H. et al. The Sequence Alignment/Map format and SAMtools. Bioinformatics 25, 20782079 (2009).

43. R Core Team. R: A Language and Environment for Statistical Computing. $R$ Foundation for Statistical Computing, Vienna, Austria ISBN 3-900051-07-0 (2017). doi:http://www.Rproject.org/

44. Xie, W. et al. Parent-independent genotyping for constructing an ultrahigh-density linkage map based on population sequencing. Proc. Natl. Acad. Sci. 107, 10578-10583 (2010). 


\section{Acknowledgements}

426 We thank Bernhard Schmid and Ueli Grossniklaus for helpful discussions and sharing

427 infrastructure. We thank Jordi Bacompte and Jacob Weiner for helpful comments on the manuscript.

428 We further acknowledge Matthias Philipp for technical support, Enrica De Luca and Nicole Ponta

429 for help with plant measurements, Matthias Furler and Dorde Topalovic for technical greenhouse

430 support. This work was supported by an Ambizione Fellowship (PZ00P3_148223) of the Swiss

431 National Science Foundation (to S.E.W). P.A.N acknowledges support by the University of Zurich

432 Priority Programme "Global Change and Biodiversity". S.E.W was also financially supported by

433 funds of the University of Zurich and the European Research Council (to Ueli Grossniklaus).

\section{Author contributions}

436 S.E.W. conceptualized and designed the research (with input from P.A.N.) and performed the 437 experiments. Both authors performed the analyses and wrote the manuscript. Both authors revised 438 and approved the final version of the manuscript.

\section{Data availability}

441 The datasets described in the paper and a functional annotation of the 86 genes within the fine-

442 mapped diversity QTL are available through the Zenodo data repository

443 (DOI:10.5281/zenodo.1254563). Sequencing data are deposited in the NCBI Short-Read Archive

444 (accession SRP149077).

445 Analysis scripts are available from the authors upon request.

\section{Competing interests}

448 The authors declare no competing financial interests. 
a
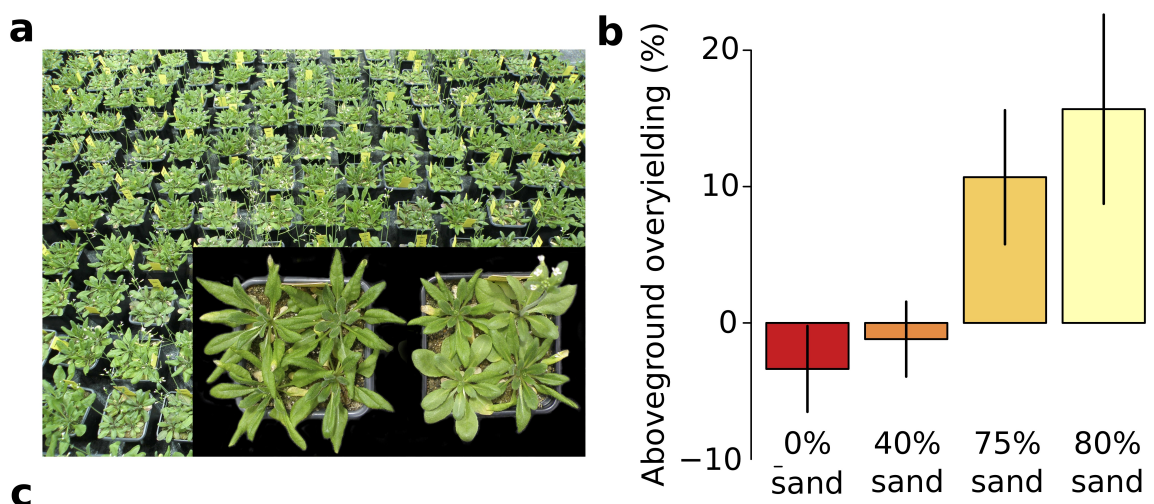

C

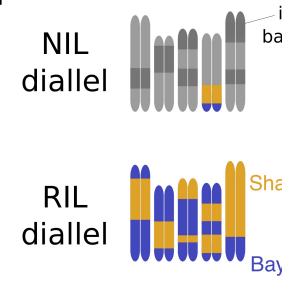

isogenic background
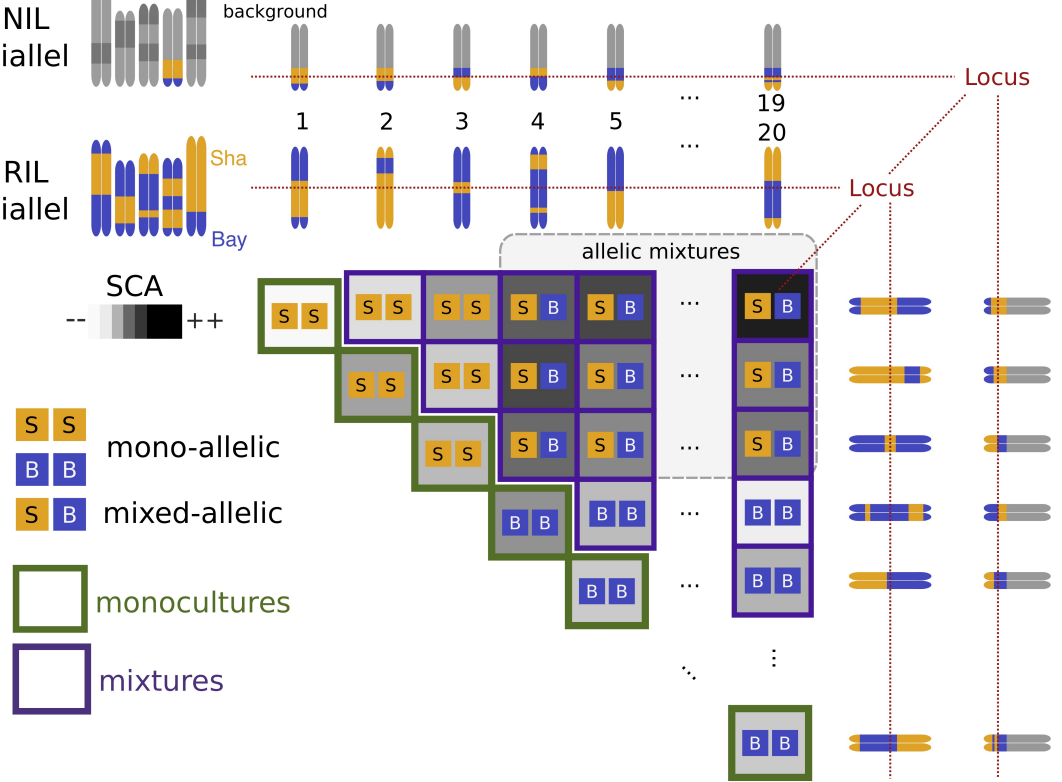

d main diallel + soil training

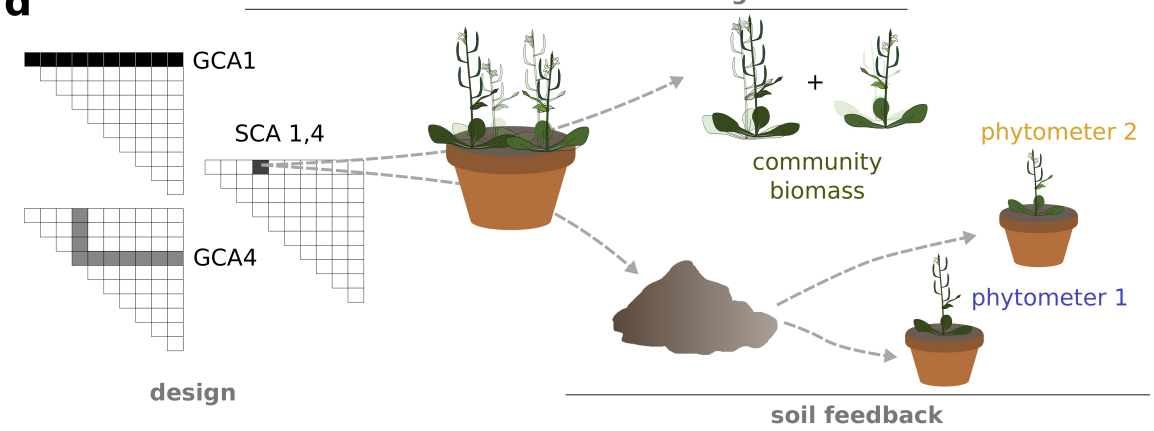

451 Figure 1 | Combining ecological concepts and genetic methods. a, Pot systems used to study diversity

452 effects in pair-wise genotype mixtures. The inset shows a Recombinant Inbred Line (RIL) monoculture

453 (left) and mixture (right). b, Net diversity effects in Bay-Sha mixtures along a peat-sand substrate

454 gradient. Error bars denote standard errors of means (s.e.m.). $n=164$ pots c, Outline of the diallel

455 design and the genotypes used throughout this paper. 18 RILs and the two parental accession, or 19 
near-isogenic lines (NILs) were each placed in competition with each other, allowing to assess i) effects of genotypic mixture (i.e. diagonal vs off-diagonal), or ii) effects of allelic mixture at a given locus across all genotype mixtures (i.e. comparing SS and BB vs. SB) on pot productivity. d, Outline of the experimental procedure used in this work. Colored labels indicate measured variables. GCA = general combining ability; SCA = specific combining ability.

461

a

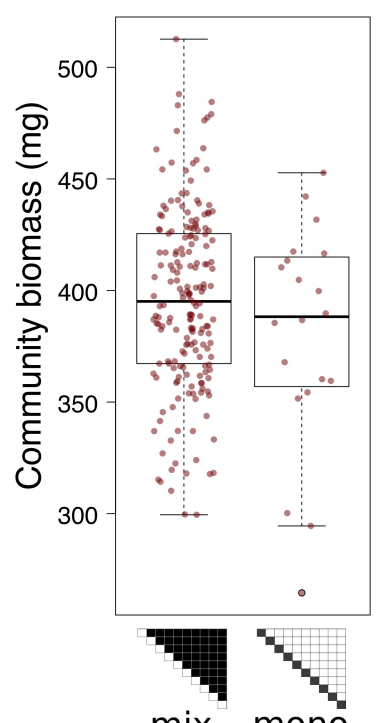

mix mono b

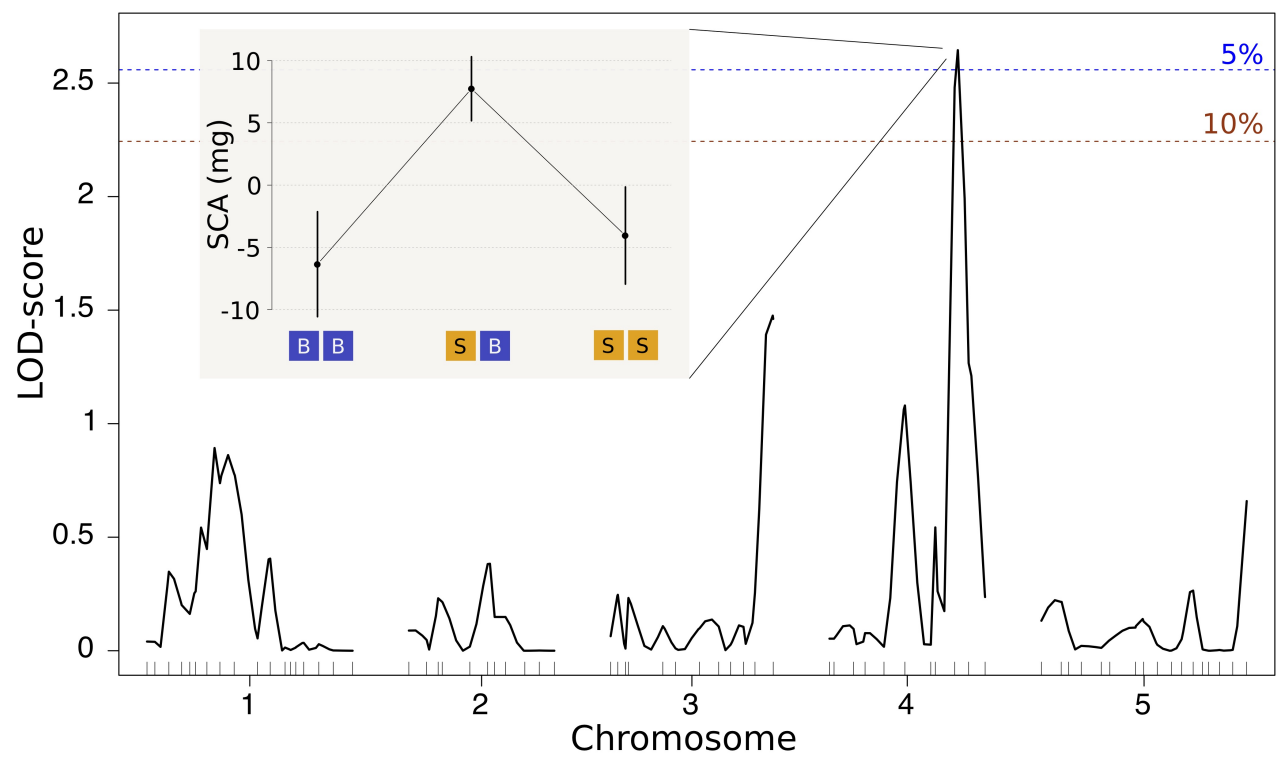

Figure 2 | Allelic diversity at a major effect locus increases community productivity. a, Pot-level productivity in dependence of community type ( $\mathrm{mix}=$ RIL mixtures vs mono = RIL monocultures), showing positive genotype mixture effects in the diallel and on sand-rich soil (values aggregated across four blocks, $\mathrm{n}=871$ pots / 210 compositions) b, Quantitative trait locus interval mapping of allelic diversity effects on specific combining ability (SCA). Vertical lines denote $10 \%$ and $5 \%$ genome-wide significance levels. The inset shows estimated SCA ( \pm s.e.m.) across genotype mixtures that exhibit different allelic compositions at marker MSAT4.9 on chromosome four. LOD: logarithm of the odd. 


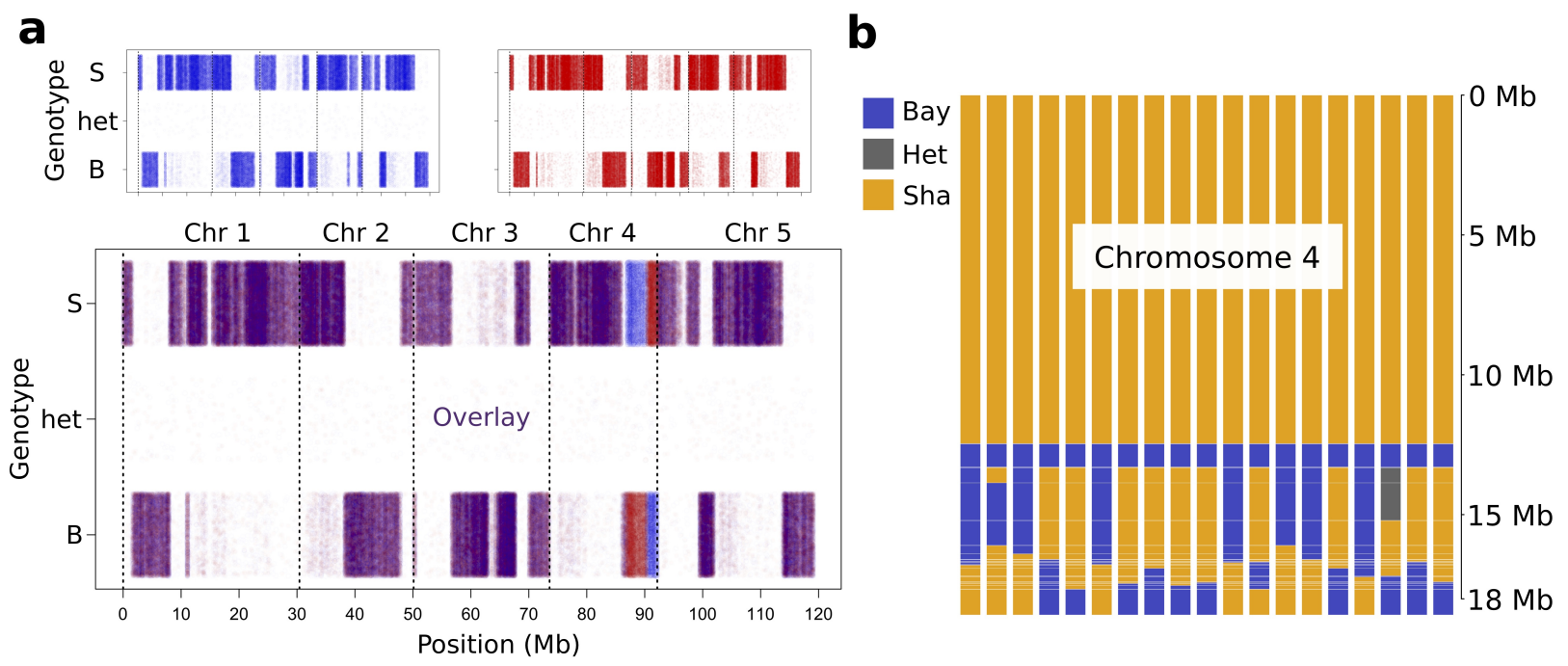

C

d
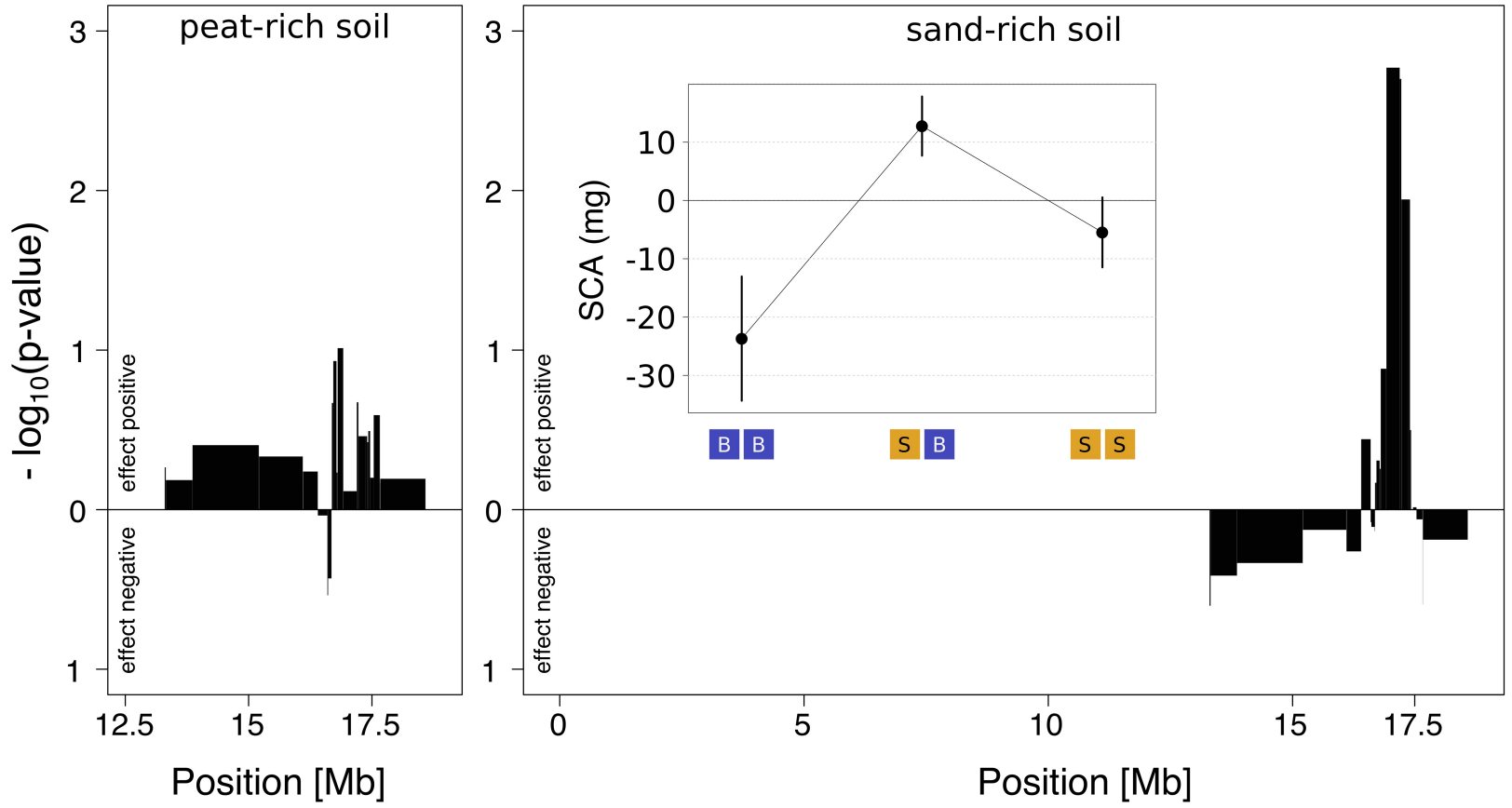

471 Figure 3 | Resolving soil $\times$ allelic diversity interactions to a single Mendelian factor. a, Re-

472 sequencing of near-isogenic lines (NILs) differing only on lower arm of chromosome four for fine-

473 mapping. Shown are genotype calls at all polymorphic sites across the genome $(\mathrm{B}=$ homozygous for the

474 Bay allele, het = heterozygous, $\mathrm{S}=$ Sha allele) in either NIL r10 (blue, top left) or NIL r96 (red, top

475 right), as well as an overlay of the two line's genotype calls (bottom). b, Reconstructed genotypes

476 across chromosome four of the 19 NILs used for fine-mapping. Each bar represents a single NIL. c, d,

477 Map of allelic diversity effects across chromosome four, on either peat-rich soil (c), or sand-rich soil

478 (d). The widths of the bars indicate the size of the regions in which no recombination events were 
a

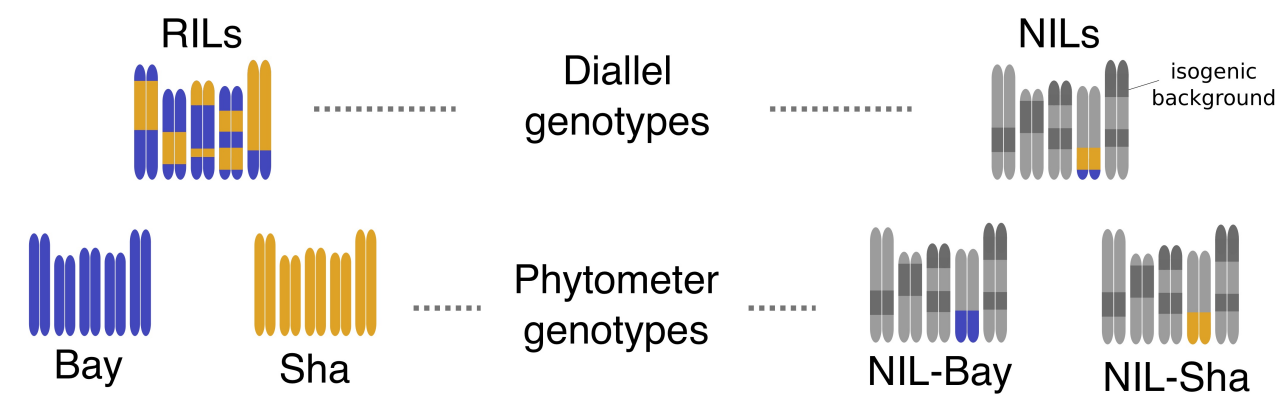

b

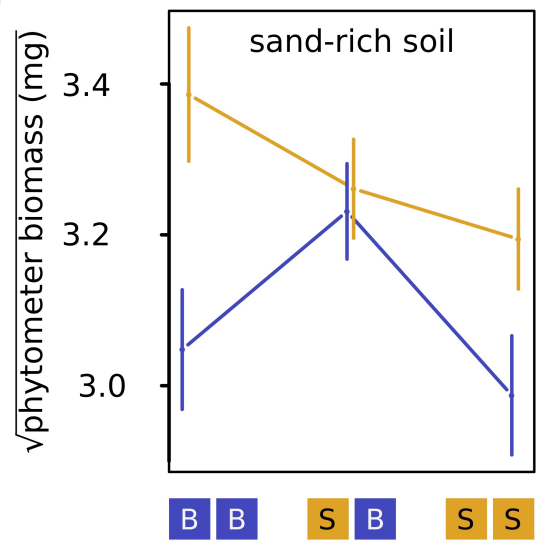

C

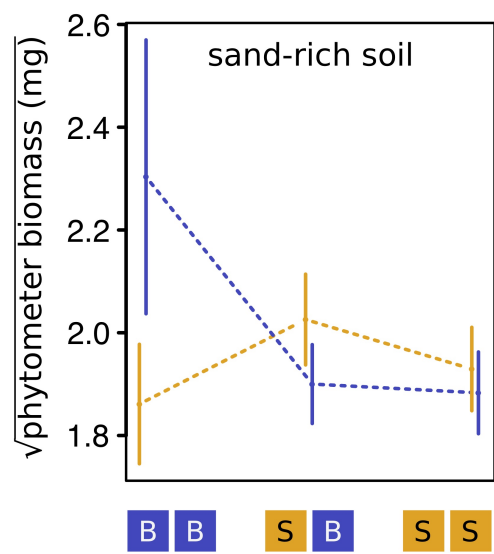

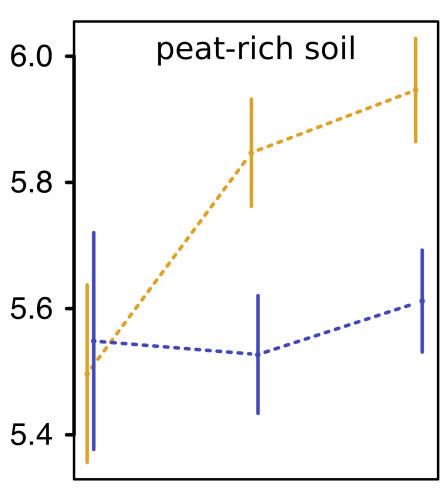

\begin{tabular}{l|ll|ll|l|l}
$B$ & $B$ & $S$ & $B$ & $S$ & $S$
\end{tabular}

Figure 4 | Allelic diversity effects persist across a generation through their soil legacy. a, Scheme of the genotype-combinations used for either the recombinant inbred lines (RILs) vs. near-isogenic lines (NILs) diallels (top), and the phytometer genotypes used in the soil-feedback phase (bottom). b, phytometer performance (Bay or Sha, mean \pm s.e.m.) on legacy soil derived from RIL mixtures with different allelic compositions at marker MSAT4.9. c, phytometer performance (NIL-Bay or NIL-Sha, mean \pm s.e.m.) on legacy soil derived from NIL mixtures with different allelic compositions at locus Chr4@16.92 on either sand-rich (left) or peat-rich (right) soil. Values were square-root transformed for analyses, $\mathrm{n}=851$ and 720 pots for RIL and NIL diallels, respectively. 


\section{Supplementary Tables}

\section{Supplementary Table 1 | Legacy effects of soils conditioned in RIL and NIL diallel}

494 experiments. Effects were quantified using two phytometers (factor 'phy'; Sha and Bay accessions

495 in RIL diallel, and near-isogenic lines bearing Sha and Bay allele at putative effect locus in NIL

496 diallel). The NIL diallel additionally was replicated on substrates differing in sand content (factor

497 'soil'). The term 'GCA' (general combining abilities) indicates average genotype-specific soil

498 conditioning effects on phytometer yields. 'SCA' (specific combining abilities) captures deviations

499 in yield from additive predictions made using GCAs. Within SCA, the following contrasts were

500 tested: GD: Genotype diversity, i.e. whether genotype monocultures differed in feedback effects

501 from two-genotype mixtures; A: allele-specific differences at marker MSAT4.9 (RIL diallel) and

502 Chr4@16.92 (NIL diallel) within genotype monocultures; AD: allele-diversity effects within

503 genotype mixtures. df and ddf indicate nominator and denominator degrees of freedom of

504 corresponding F-tests. *** $\mathrm{P}<0.001 ; * * \mathrm{P}<0.01 ; * \mathrm{P}<0.05 ;(*) \mathrm{P}<0.1$; n.s. not significant.

505

\begin{tabular}{|c|c|c|c|c|c|c|}
\hline \multirow{2}{*}{$\begin{array}{l}\text { Terms and } \\
\text { contrasts }\end{array}$} & \multicolumn{3}{|c|}{ RIL diallel } & \multicolumn{3}{|c|}{ NIL diallel } \\
\hline & df & Denominator: ddf & Signif. & df & Denominator: ddf & Signif. \\
\hline GCA & 18 & comp: 167 & $\left({ }^{*}\right)$ & 18 & comp: 168 & n.s. \\
\hline \multicolumn{7}{|l|}{ SCA } \\
\hline GD & 1 & comp: 167 & $\left({ }^{\star}\right)$ & 1 & comp: 168 & n.s. \\
\hline A & 1 & comp: 167 & n.s. & 1 & comp: 168 & n.s. \\
\hline AD & 1 & comp: 167 & n.s. & 1 & comp: 168 & n.s. \\
\hline Phy $\times$ GCA & 18 & phy $\times$ comp: 166 & n.s. & 18 & phy $\times$ comp: 168 & * \\
\hline \multicolumn{7}{|l|}{ Phy $\times$ SCA } \\
\hline Phy × GD & 1 & phy $\times$ comp: 166 & n.s. & 1 & phy $\times$ comp: 168 & n.s. \\
\hline Phy $\times A$ & 1 & phy $\times$ comp: 166 & n.s. & 1 & phy $\times$ comp: 168 & n.s. \\
\hline Phy $\times$ AD & 1 & phy $\times$ comp: 166 & ** & 1 & phy $\times$ comp: 168 & * \\
\hline Soil $\times$ GCA & & & & 18 & soil $\times$ comp: 150 & n.s. \\
\hline \multicolumn{7}{|l|}{ Soil $\times$ SCA } \\
\hline Soil × GD & & & & 1 & soil × comp: 150 & n.s. \\
\hline Soil $\times A$ & & & & 1 & soil × comp: 150 & n.s. \\
\hline Soil $\times A D$ & & does not & & 1 & soil $\times$ comp: 150 & n.s. \\
\hline Phy $\times$ Soil $\times$ GCA & & apply & & 18 & phy $\times$ soil $\times$ comp: 146 & n.s. \\
\hline \multicolumn{7}{|l|}{ Phy $\times$ Soil $\times$ SCA } \\
\hline Phy $\times$ Soil $\times$ GD & & & & 1 & phy $\times$ soil $\times$ comp: 146 & $* * \star$ \\
\hline Phy $\times$ Soil $\times A$ & & & & 1 & phy $\times$ soil $\times$ comp: 146 & n.s. \\
\hline Phy $\times$ Soil x AD & & & & 1 & phy $\times$ soil $\times$ comp: 146 & n.s. \\
\hline
\end{tabular}


bioRxiv preprint doi: https://doi.org/10.1101/264960; this version posted August 28, 2018. The copyright holder for this preprint (which was not certified by peer review) is the author/funder, who has granted bioRxiv a license to display the preprint in perpetuity. It is made available under aCC-BY-NC-ND 4.0 International license.

507 Supplementary Table 2: PCR markers used in this study.

\begin{tabular}{|l|l|l|l|l|}
\hline Primer & Assay & Sequence & Predicted & Pred. fragment \\
location & (Sha/Bay) \\
\hline SW-182 & ShaBa5 & ACGTATTTCGATGTATGGTCCTTG & Chr4: 16044156 & $550 / 664$ \\
\hline SW-183 & & $\begin{array}{l}\text { TCACGTGAATCGTATTCGTTGAAG } \\
\text { CTTCTCCGCTTCAACCTCTGC }\end{array}$ & Chr4: 17709750 & $600 / 632$ \\
\hline SW-184 & ShaBa6 & AATCCAGGATTCAGAGTTGCTTTC & & \\
\hline SW-185 & & TTGATTAGGGCTACGAGGATAAGG & Chr4: 16707214 & $408 / 609$ \\
\hline SW-188 & ShaBa8 & GAGTCTATTAATTATGCTTGGTGC & & \\
\hline SW-189 & & & & \\
\hline
\end{tabular}




\section{Supplementary Figures}
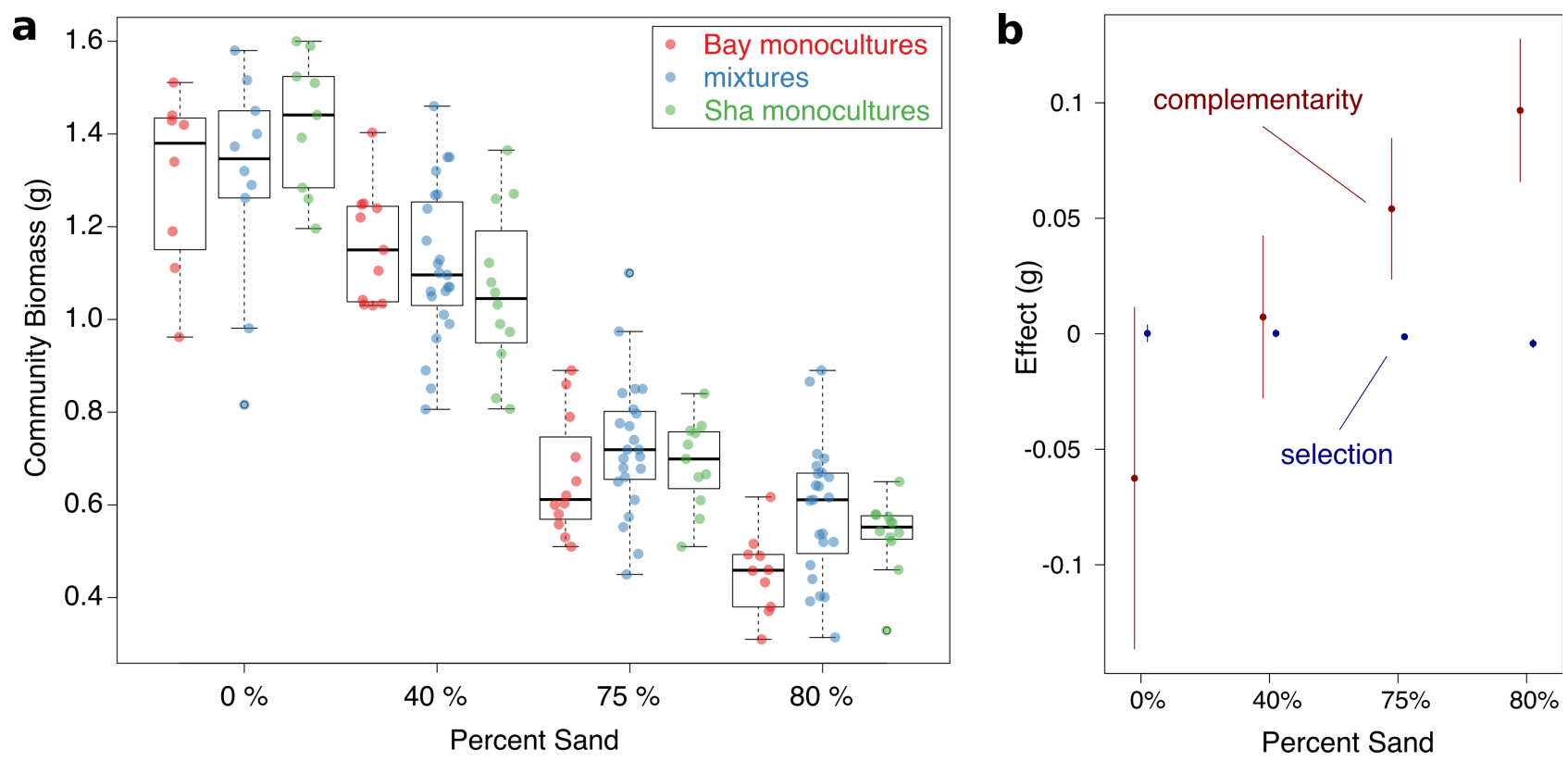

\section{Supplementary Figure 1 | Productivity and complementarity in Bay-Sha mixtures across a}

513 peat-sand gradient. a, Pot-level biomass measurements of Sha and Bay monocultures or pair-wise

514 mixtures along a sand/peat substrate gradient. $\mathrm{n}=164$ pots $\mathbf{b}$, Complementarity and selection

515 effects calculated according to the additive partitioning ${ }^{1}$ method along the substrate gradient. Error

516 bars denote s.e.m.

517 
a

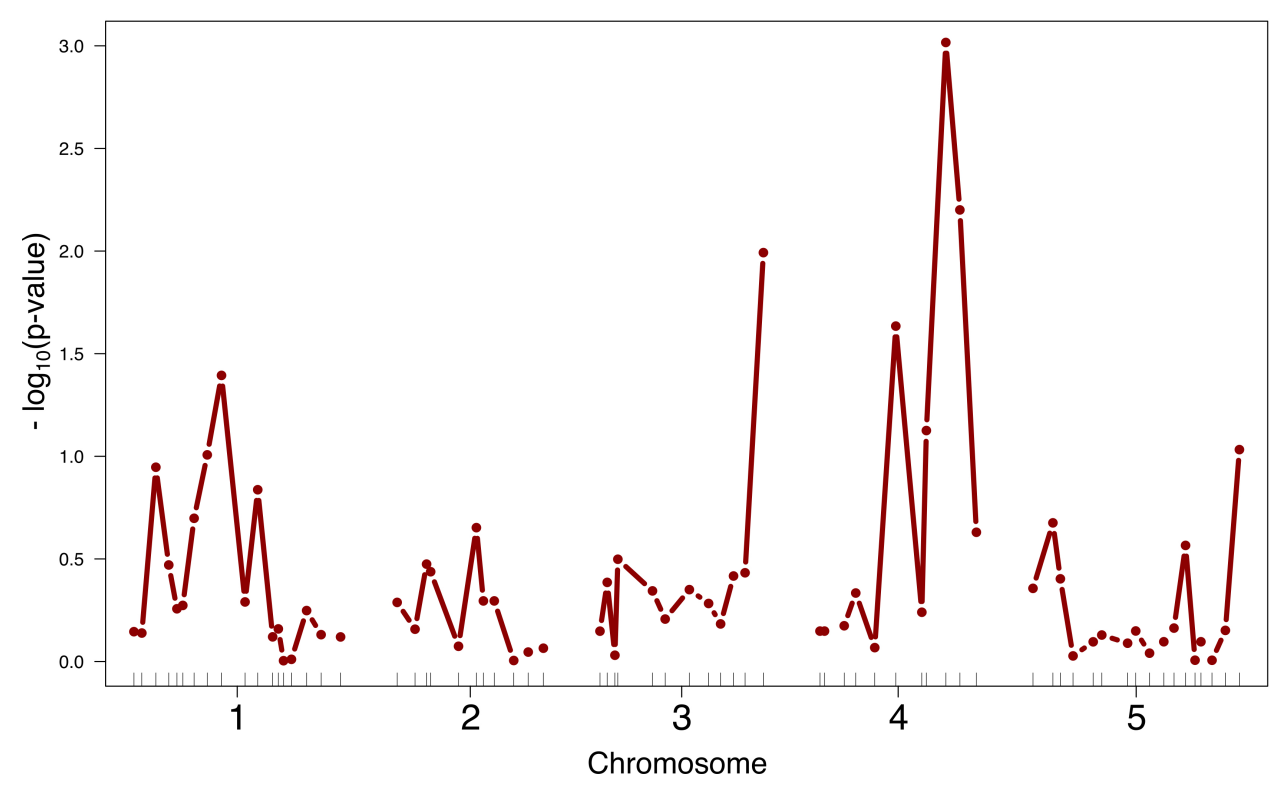

b

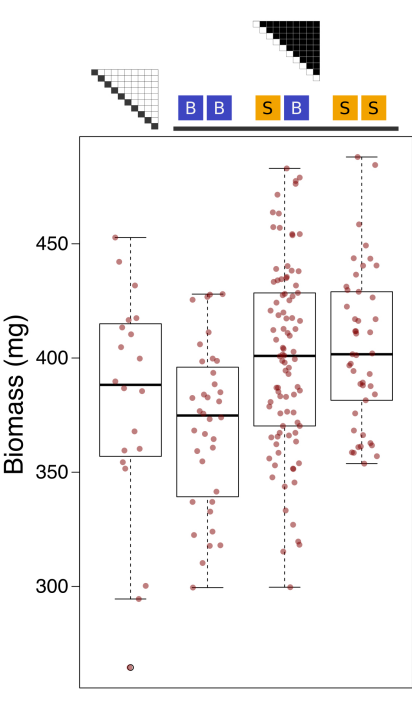

519 Supplementary Figure 2 | A major effect locus driving complementarity between genotypes. a, QTL mapping of SCA variation across allelic diversity levels using a marker regression technique

521 by contrasting SCAs of mono-allelic RIL mixtures (BB and SS) with bi-allelic mixtures (BS). b,

522 Pot-level productivity of each genotype composition (average of four blocks) in dependence of

523 allelic composition at marker MSAT4.9. $\mathrm{n}_{\text {composition }}=210$ 

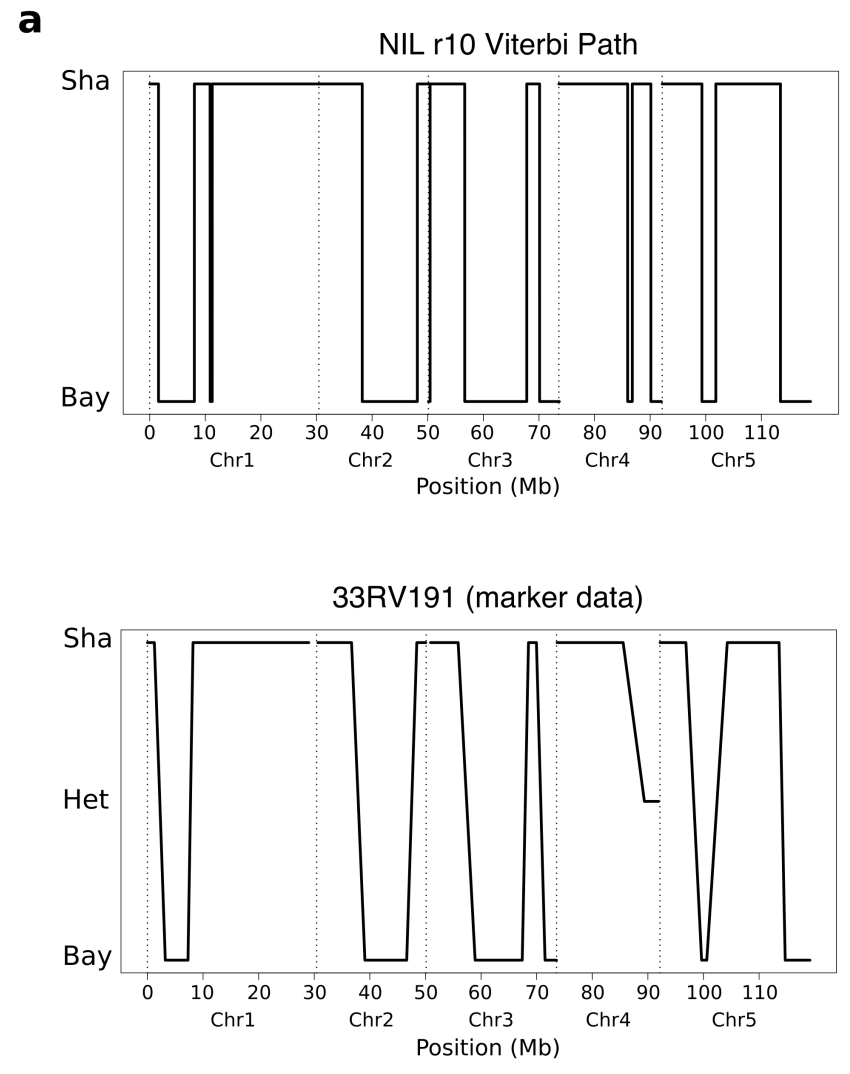

b
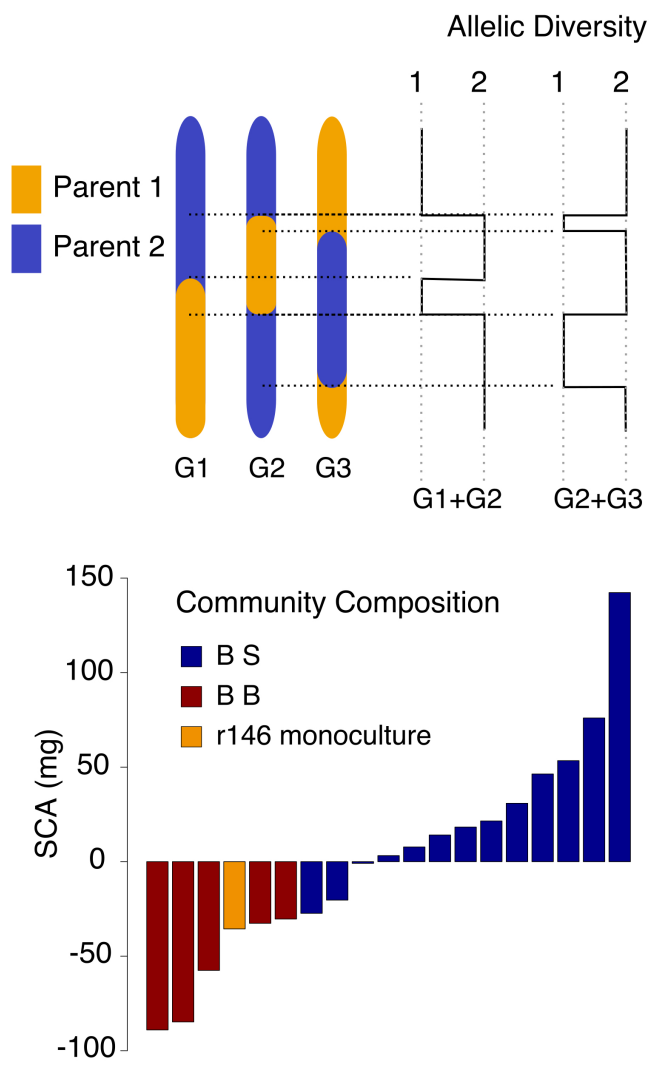

524 Supplementary Figure 3 | Fine-mapping and persistence of allelic diversity effects in near-

525 isogenic line. a, The comparison of the reconstruced genotype of NIL r10 (HMM Viterbi-path

526 across all chromosomes, homozygous on lower arm of chromosome 4) in comparison to publicly

527 available molecular marker-based genotyping data of the ancestral line from which it was derived

528 (heterozygous on lower arm of chromosome 4) - showing a high degree of congruence between the

529 re-constructed genotype based on whole-genome resequencing and the marker data. b, Schematic

530 outline of a possible cause of the high mapping resolution achieved through the diallel design. A

531 major advantage of the design is the joint dependency of community-level allelic diversity on

532 recombinations within and between recombinant inbred lines, such that mapping resolution

533 increases very quickly. c, Extreme example of SCA variation across genotype mixtures all

534 containing one specific genotype (NIL r146, homozygous for the Bay-allele at locus Chr4@16.92), 
535 either in combinations with NILs carrying the Sha-allele (dark blue bars) or the Bay-allele (dark red

536 bars). Shown are the data from sand-rich soil only.

a

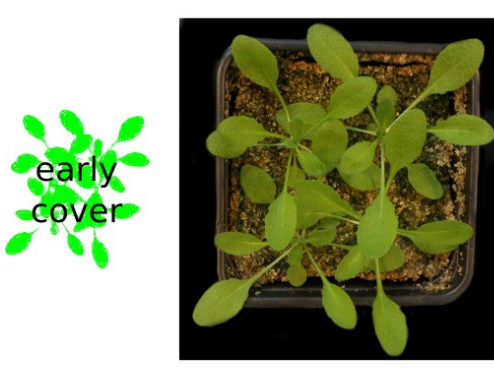

leaf disease

bolt length

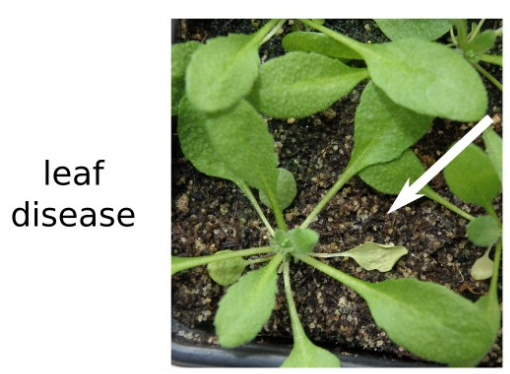

b

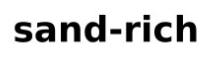

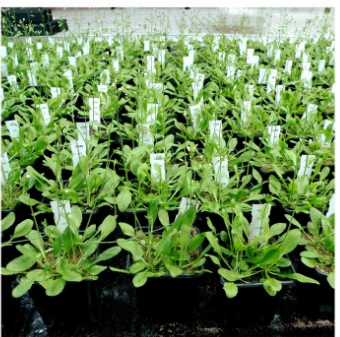
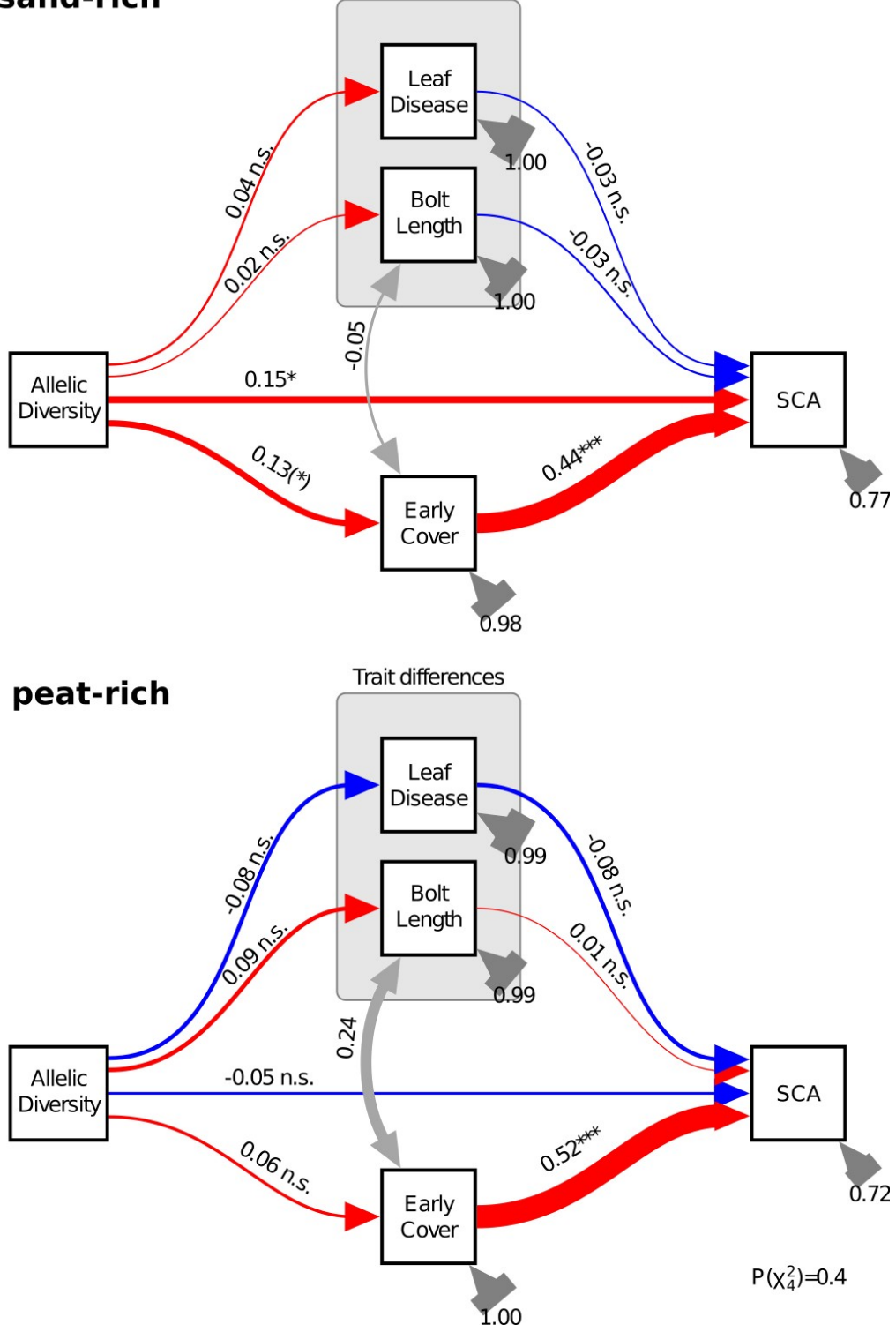

\section{Supplementary Figure 4 | Observed above-ground trait variation does not explain} overyielding of diallelic mixtures. a, Traits measured in the NIL diallel as proxy for productivity

542 (early growth projected leaf cover), disease susceptibility (leaf disease at 30 days after sowing) or

543 phenology (bolt length at 35 days after sowing) b, Path diagram of multi-group structural equation 
544 model showing direct effects of allelic diversity at locus Chr4@16.92 on SCA on sandy but not on

545 peat soil. Red and blue arrows show positive and negative standardized path coefficients,

546 respectively. n.s. $=$ not significant. $(*)=\mathrm{p}<0.1 ; *=\mathrm{p}<0.05 ; * *=\mathrm{p}<0.01$.

548
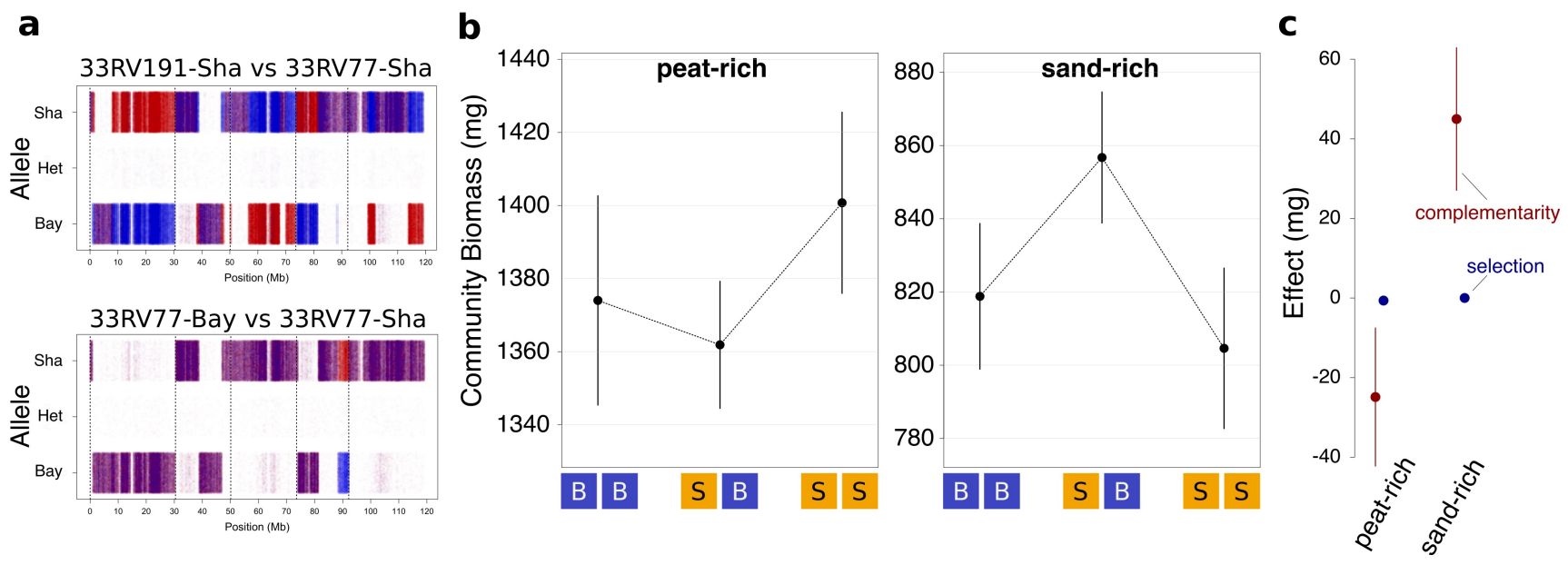

549 Supplementary Figure 5 | Soil-dependent effect of allelic diversity on overyielding and statistical complementarity and selection effects ${ }^{\mathrm{S1}}$ in an independent near-isogenic

background. a, Overlay of genotype calls at all polymorphic sites across the genome (Sha and Bay = homozygous for respective allele, het $=$ heterozygous) of lines used for a mendelization. Top: a comparison of the genetically independent backgrounds 33RV191-Sha (in red, this background was used for the fine-mapping shown in Fig. 3) and line 33RV77-Sha (in blue) is shown. At the bottom, a comparison of the near-isogenic lines 33RV77-Sha (red) vs 33RV77-Bay (blue), the two lines that were used in the experiment shown in b and c. Purple regions depict overlap of genotype calls, vertical lines separate the different chromosomes. b, Mean \pm s.e. of final aboveground biomass of communities

558 consisting either of 33RV77-Sha plants only (BB), of mixed communities (BS), or of communities consisting of 33RV77-Bay plants only (SS) on either peat-rich (left) or sand-rich (right) soil. c,

560 Complementarity and selection effects sensu Loreau and Hector calculated from the experiment 561 shown in $b$. 


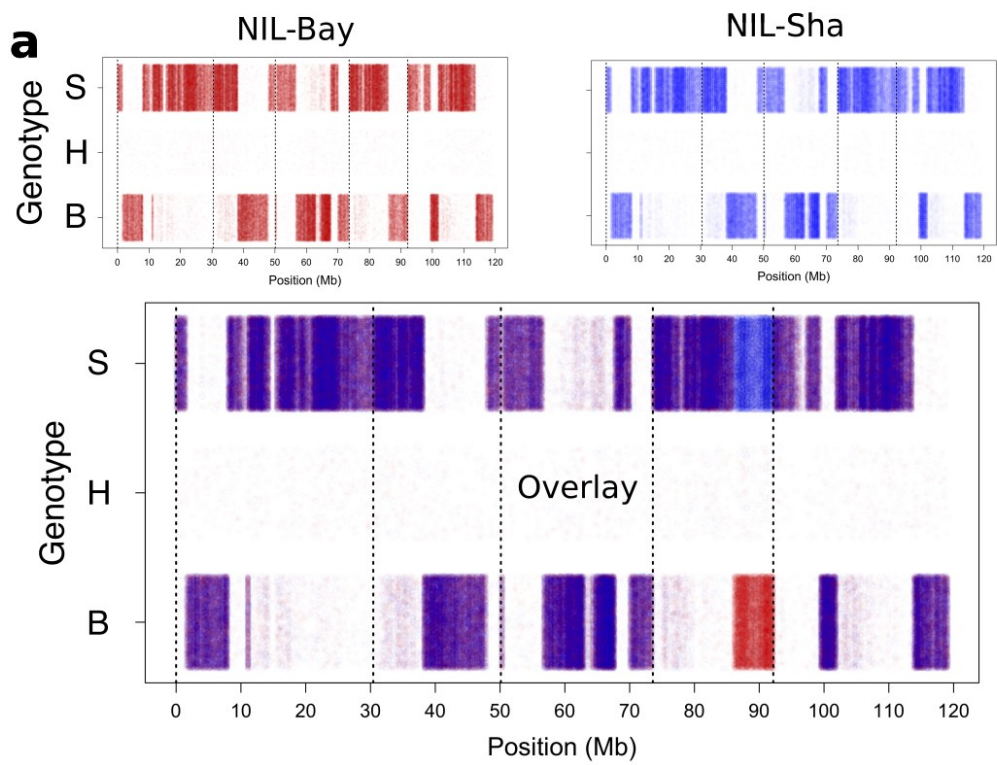

b
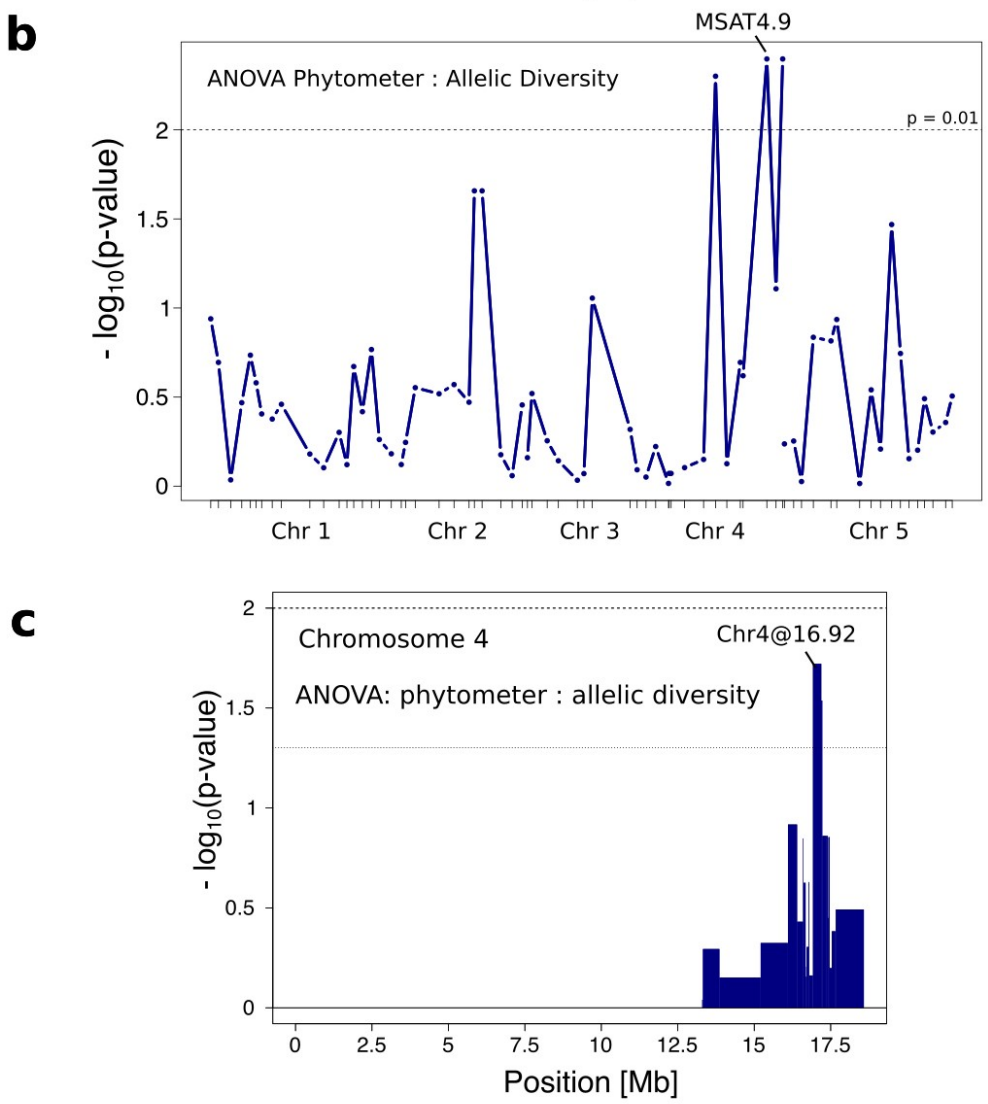

563 Supplementary Figure 6 | Soil feedback experiments. a, Genotype calls at all polymorphic sites

564 across the genome $(\mathrm{B}=$ homozygous for the Bay allele, $\mathrm{H}=$ heterozygous, $\mathrm{S}=$ homozygous for the

565 Sha allele) obtained by genome re-sequencing phytometer genotypes NIL-Bay (33RV191-Bay, top

566 left, red) and NIL-Sha (33RV191-Sha, top right, blue). The overlay of the genotype calls of both 
567 lines (bottom) confirms that these lines are isogenic but for variation on lower arm of chromosome

568 four. The two phytometers were employed on soil derived from the NIL diallel. b, c, QTL mapping

569 by marker regression of phytometer-specific responses to soil legacy of previous generation allelic

570 diversity (i.e. allele-diversity $\times$ phytometer $[\mathrm{Phy} \times \mathrm{AD}]$ interaction in Supplementary Table 1, but in a

571 model without adjusting for diallel pot biomass). The mapping of diversity effects through such

572 influences on soil legacy could have been applied to the identification and fine-mapping of the same

573 major effect locus, without ever measuring biomass productivity (albeit with slightly relaxed

574 statistical criteria). Shown are negative $\log _{10}$-transformed P-values for each marker position in the

575 RIL (b) or genotype block in the NIL (c) diallels.

\section{Supplementary Discussion}

578 As outlined in Figures 1 and 4, and as described in the Methods, we performed two soil-feedback

579 experiments to test whether allelic diversity effects extend across generations. We accounted for

580 potential effects explainable by variation in plant productivity during the soil training phase (e.g.

581 nutrient draw-down or environmental correlations) in linear models (terms diallel biomass and

582 diallel biomass $\times$ Soil as described in the Methods section). Significantly different soil conditioning

583 through allelic diversity, as assessed by phytometer performance in a next growing period, was

584 interpreted as an "extended phenotype"\$2 (i.e. a legacy of allelic mixture that persists through time

585 in the soil, even after removal of the original plant communities). These soil factor-mediated allelic

586 legacy effects interact with phytometer genotype, giving rise to phytometer-specific responses (term

587 “Phy×AD” in Supplementary Table 1).

588 As we emphasize, the specific mechanisms underlying both soil training and phytometer responses

589 await further experiments, since the response patterns (Fig. 4) do not allow for a simple mechanistic

590 model. One reason why it is difficult to infer specific mechanisms (e.g. specific soil factors, and

591 their interactions with genetic variants) is the number of variables that differed between the two 
experiments because of constraints on experimental design: 1) environmental conditions (different calendar dates, different batches of soil); 2) genetic variation in the two diallel population (RILs vs

594 NILs), potentially resulting in different effects of epistasis; and 3) phytometer genotypes (parental 595 lines vs NILs).

596 In the RIL diallel, we used the two parental accession Bay and Sha as phytometers and a split-plot 597 design to test for differential responses of these phytometer to soil conditioning. After the NIL 598 diallel, we used two near-isogenic phytometer (33RV191-Bay and 33RV191-Sha) as phytometers in 599 a similar test for differential responses to soil conditioning. Naively, one might expect 600 approximately congruent responses of the phytometers carrying the same alleles at the diversity 601 locus under question, i.e. the parental genotype Bay in the RIL diallel (Figure 4 b, yellow lines) and 602 the NIL-Bay genotype in the NIL diallel (Figure $4 \mathrm{c}$, yellow lines) might respond similarly to the 603 legacy of allelic diversity. However, this was not the case. For example, the Bay genotype grown on 604 RIL diallel soil responded positively to conditioning by allelic diversity, whereas the NIL-Bay 605 genotype in the NIL diallel responded somewhat negatively to conditioning by allelic diversity. It is noteworthy that the pattern found for Bay on RIL diallel soil is opposite to what would be expected if soil legacy was driven by a simple productivity-related depletion of resources in the conditioning phase; this suggests that allelic diversity alleviates a negative soil factor (e.g. inhibits enemies), or promotes a positive factor (e.g. mutualistic organisms).

Despite differences, the RIL and NIL soil-feedback experiments nevertheless convincingly

611 demonstrate i) that allelic diversity effects extend across time through soil conditioning, and ii) that

612 phytometer genotype determines the response to such conditioning (phytometer $\times$ allelic diversity 613 interaction, both experiments, see Table S1). The fact that allelic diversity effects within a 614 community and allele-specific legacy effects on individuals separated in time map to the same locus suggests to us that both are related to similar mechanisms. 
bioRxiv preprint doi: https://doi org/10.1101/264960; this version posted August 28, 2018. The copyright holder for this preprint (which was

not certified by peer review) is the author/funder, who has granted bioRxiv a license to display the preprint in perpetuity. It is made available under aCC-BY-NC-ND 4.0 International license.

\section{Supplementary References}

618

619 1. Loreau, M. \& Hector, A. Partitioning selection and complementarity in biodiversity

$620 \quad$ experiments. Nature 412, 72-76 (2001).

621 2. Dawkins, R. The Extended Phenotype. (Oxford University Press, 1982).

622 